\title{
MR IMAGING SPECTRUM OF JAPANESE B ENCEPHALITIS IN PEDIATRIC AGE GROUP
}

Pronami Borah ${ }^{1}$, Dhaval D. Dhingani' ${ }^{2}$ Rudra K. Gogoi ${ }^{3}$

\author{
${ }^{1}$ Assistant Professor, Department of Radiodiagnosis, Assam Medical College \& Hospital, Dibrugarh. \\ ${ }_{2}^{2}$ Post Graduate Trainee, Department of Radiodiagnosis, Assam Medical College \& Hospital, Dibrugarh. \\ ${ }^{3}$ Professor, Department of Radiodiagnosis, Assam Medical College \& Hospital, Dibrugarh.
}

ABSTRACT: BACKGROUND: Japanese B encephalitis is an important public health problem, mainly in the districts of upper Assam. MRI Brain with its conventional T2/FLAIR sequences as well as DWI \& ADC mapping can clearly depict the site \& extent of involvement of different parts of the brain along with any associated coinfections. Especially DWI sequence shows findings that closely follows the pathological changes of the encephalitic process.

OBJECTIVE: To evaluate Japanese B encephalitis in pediatric age group with MRI Brain.

METHODS: It is a retrospective descriptive observational study carried out on AES cases referred to our department from Pediatric department for MRI Brain during January- September 2015. All cases were evaluated as per following protocol. Axial T1weighted (T1-W) \& T2-weighted (T2-W), axial \& coronal fluid-attenuated inversion recovery pulse image (FLAIR), axial GRE/SWI and axial Diffusion-weighted imaging (DWI) with ADC (Apparent diffusion coefficient) sequences were taken. In cases where contrast study was indicated, post contrast T1-W fat saturated (T1FS) sequence in axial, coronal and saggital planes were taken. Serological tests, i.e., MAC ELISA and VNT, for the diagnosis of JE were done by using paired blood \& CSF samples.

RESULTS: Out of the 24 cases referred for MRI evaluation, JE was confirmed serologically in 21 patients. Most of the cases showed symmetrical T2/FLAIR hyperintensity in bilateral Thalami \& Substantia nigra.

CONCLUSION: Japanese B Encephalitis is the most common \& important cause of AES in this region of Upper Assam, especially in pediatric age group mainly in the rainy season. MRI could detect the disease process as efficiently as that of serological and CSF studies along with its coinfections.

KEYWORDS: MRI, JE, Pediatric age.

HOW TO CITE THIS ARTICLE: Pronami Borah, Dhaval D. Dhingani, Rudra K. Gogoi. "MR Imaging Spectrum of Japanese B Encephalitis in Pediatric Age Group." Journal of Evolution of Medical and Dental Sciences 2015; Vol. 4, Issue 93, November 19; Page: 15863-15867, DOI: $10.14260 /$ jemds/2015/2302.

INTRODUCTION: BACKGROUND: Japanese encephalitis is an important public health problem in Assam, mainly in the districts of upper Assam. The high case fatality rate (20\%-30\%) and frequent residual neuropsychiatric damage in survivors (50\%-70\%) make JE a major public health problem.(1) Japanese encephalitis virus (JEV) has caused sporadic epidemics in Assam since 1976.(2) Recurrent epidemics of JE in different magnitudes occur in this particular region of Assam during the months of July to October every year.(3)

In our institute during the year 2015, 194 cases of AES were admitted in the Dept. of Pediatrics till the month of September, out of which 69 cases were diagnosed to be JE positive(35\%) and others were non JE AES. Out of these 194 AES patients 36 patients were expired (18\%), out of which 13 patients were JE positive. Mortality due to AES has been in decline due to better hospital facility, but there is concomitant rise in the number of patients with sequelae. Around $30 \%$ of the AES survivors develop frank motor deficit.(4) and 20\% survivors have cognitive and language impairments. (5)

According to U.K. Misra et al.,1-4 weeks after the onset of the disease, movement disorders are seen usually.(6)

Financial or Other, Competing Interest: None.

Submission 03-11-2015, Peer Review 04-11-2015,

Acceptance 11-11-2015, Published 18-11-2015.

Corresponding Author:

Dr. Pronami Borah,

Assistant Professor,

Department of Radiodiagnosis,

Assam Medical College \& Hospital,

Dibrugarh.

E-mail: pronamiborah@yahoo.in

DOI:10.14260/jemds/2015/2302.
According to one study, about half of the patients who were classified as good recovery group have more subtle sequelae such as learning difficulties, behavioral problems and subtle neurological signs.(7) To our knowledge few studies are available in brain MRI evaluation of AES cases in pediatric age group in this JE endemic region. Through this study we would like to focus on the role of Brain MRI for evaluation of AES in pediatric age group.

OBJECTIVES: The aim of this study was to evaluate brain MRI findings of Japanese B encephalitis in pediatric age group.

METHODS: All the AES cases of either sex and up to eighteen years of age, referred to our department for MRI Brain, were included in the study. The study was carried out during January - September 2015. AES was defined according to WHO clinical case definition.(1) as a person of any age, at any time of year with the acute onset of fever and a change in mental status (Including symptoms such as confusion, disorientation, coma or inability to talk) and or new onset of seizure (Excluding simple febrile seizure).

Other early clinical findings may include an increase in irritability, somnolence or abnormal behaviour; greater than that seen with simple febrile illness. ${ }^{(8,9)}$ Pretested and predesigned proforma was used for this research purpose. The history and clinical examination of the patients was documented. After taking written consent from the patient or from the legal guardian (In case patient was unable to give), MRI brain with or without contrast was performed. MRI of the Brain and Spine (If required) were carried out on a $1.5 \mathrm{~T}$ Machine. 
The sequences taken were T1-weighted image (T1-W), T2-weighted image (T2-W), fluid-attenuated inversion recovery pulse image (FLAIR), GRE/SWI, Diffusion-weighted imaging (DWI) with ADC and post contrast T1FS sequence if required. FLAIR sequence was taken in both axial and coronal plane. Other conventional spine echo sequences were taken in only axial plane. Post contrast MP rage sequence was also taken. The diagnosis was confirmed by MAC ELISA TEST and VNT as per standard protocol at RMRC, near Dibrugarh.

Serological test for diagnosis of JEV infection was carried out by JE MAC ELISA kit (NIV, Pune). Differential ELISA for WN and subsequently VNT was done to exclude the West Nile infections because the NIV JE ELISA kit being flavivirus specific does not differentiate between JEV and WNV infections. Convalescent serum samples were tested for four fold rise in antibody titers against the ELISA diagnosed viral infection as a confirmatory test. Based on well described imaging features.(10) diagnosis of NCC was made on neuroimaging studies in cases that have this parasitic infestation along with AES.

RESULTS: This is a retrospective descriptive observational study carried out on 24 AES cases referred to our department from Pediatric department for MRI Brain during January September 2015. Out of these cases JE was confirmed in 21 cases. One case was of metabolic encephalopathy and three cases had Tubercular Meningitis. Out of the confirmed JE cases, 3 cases (14.3\%) did not reveal any abnormal T2/FLAIR hyperintensity or DWI restriction on MRI. Out of 21 cases, $11(52.4 \%)$ were girls and $10(41.65)$ were boys.

Although the disease was seen throughout the year in a varying pattern, similar to the Ram et al.,(11) most of the cases occur during the months of July - September and they did not have a history of travelling outside their districts.

Most of the patients presented with seizures and signs of meningeal irritability along with fever and altered sensorium.

On MRI the lesions were hypointense on $\mathrm{T} 1$ and hyperintense on T2/FLAIR.

7 cases $(33.3 \%)$ were presented in the early phase of the disease showed DW restriction with low ADC values signifying acute encephalitic process, 3 cases (14.3\%) were presented in the sub-acute phase of the disease showed DW restriction, but high $\mathrm{ADC}$ value (shine through effect) signifying sub-acute encephalitic process and later on in the chronic phase of the disease process DW restriction was not seen. GRE sequence showed blooming signifying hemorrhage in only one case.

Lesions were seen in the Thalamus, Substantia nigra and basal ganglia. Thalamic lesions were bilaterally symmetrical in $33.3 \%$ of cases and asymmetrical in $28.6 \%$ cases. Coronal FLAIR sequence is very important in this regard, as it is easier to compare bilateral lesions for symmetry in coronal sequence. Bleed was seen in only one case. Necrosis was seen in three cases. Post contrast enhancement was seen in only one case. Seven patients showed bilateral basal ganglia involvement.

Substantia nigra involvement was seen in $11(57.9 \%)$ cases. Bilateral thalamic involvement was seen in $14(66.7 \%)$ cases.
Lesions were also seen in the frontal lobe in four cases (19.04\%), Insular cortex in two cases (Fig: 4 A red arrow and $5 \mathrm{D}$ white arrow), Occipital lobe in one case, Temporal lobe in one case and Hippocampal involvement was seen in one case (Fig: 5 B red star). Associated Thalamic and Substantia nigra involvement were seen in all these cases. Coinfection with NCC was seen in ten patients (47.6\%).

DISCUSSION: Many conditions might mimic viral encephalitis hence to exclude a structural lesion cranial imaging along with CSF studies are crucial.(12) MRI shows structural alterations and extension of involvement which helps to identify the patients with risk of impending CNS abnormalities and will help in initiation of early treatment accordingly.(13) The most consistent finding in JE is bilateral thalamic lesions on MR imaging, which was seen in $66.7 \%$ case in our study.

Lesions are also noted in the Substantia nigra, basal ganglia, brain stem, cerebral cortex and white matter.(14,13,15,16,17) In most of the cases MRI with T2-W and FLAIR sequences can detect the lesions of viral infection within the first $48 \mathrm{hrs} .{ }^{(18,19)}$ MR appearance of encephalitis with DWI closely relates pathological changes that occur following a viral invasion.(19) In acute stage there is cytotoxic edema, leading to restricted diffusion and Low ADC value (Apparent diffusion coefficient).(13,20,21) Again encephalitic lesions can be seen more clearly and broadly in DWI than other conventional sequences in early stage and with conventional sequences DWI can be used to determine the phase of the disease.(22,21)

In late acute and sub-acute stages the perivascular cuffing and vasculitis diminishes and so the diffusion restriction decreases and ADC value rises.(23) This stage now becomes vasogenic edema and this is responsible for the T2W hyperintensities. Significant direct correlation between ADC value and disease duration is seen in patients with Japanese Encephalitis.(23) So a pattern of persistent cytotoxic edema suggests fulminant necrotizing changes and poor prognosis, whereas a pattern of vasogenic edema represents mild pathological changes and better prognosis. $(20,23)$

Prevalence of coinfection with NCC was seen more in our study; 10 out of 21 patients $(47.6 \%)$ had NCC and these patients also showed areas of necrosis at the time of first scan. This out numbers the prevalence of coinfection with NCC in adult age group as seen in other studies.(24) Intensity of lesions in the Thalamus was seen more on the side of neurocysticerci (Fig: 1 A-D)as that seen in Handique et al., and Singh P et al., $(24,25)$ Asymmetry of the JE lesions were noted similar to other studies done in adult population. $(24,25)$ This supports the possibility of facilitation of JE infection or neurotropic viral infection by this larval infestation even in children.

But in one patient where the NCC focus in colloidal state, i.e., when the infective focus was in early state, not ruptured, bilateral symmetry of the encephalitic lesions was observed (Fig: 2 A-E). In this region of Upper Assam pig rearing and consumption of pig meat is more. This might also increase the frequency of infection, both intestinal helminthiasis and viral infection. As pigs are the amplifying host in JE and intermediate host in cysticercosis. 
Again other factors that might predispose children to both larval and neurotropic viral infection more than adult are, children are more focused to dirty environment while playing outside, poor personal hygiene, poor socio-economic conditions, malnutrition along with immunological factors and immature CNS or neuronal plasticity. ${ }^{(24,26)}$ Severity of JE was more in patients having coinfection with NCC.

CONCLUSION: Japanese B Encephalitis is the most common and important cause of AES in this region of Upper Assam in pediatric age group, mainly in the rainy season. MRI could detect the disease process as efficiently as that of serological and CSF studies along with its coinfections. MRI being a multiplanner imaging, specially coronal FLAIR with conventional and special sequences like DWI and GRE sequences can differentiate accurately different etiologies of AES specially in pediatric age group.

\section{REFERENCES:}

1. Japanese encephalitis surveillance standards* January 2006, * WHO-recommended standards for surveillance of selected vaccine-preventable diseases WHO/V\&B/03.01;pg 46.

2. Siraj A. Khan, P Dutta, A M. Khan, P. Chowdhury, J. Borah, P Doloi, and J Mahanta, First evidence of West Nile virus infection in Assam: North East India.Emerg.Infect.Dis.2011.17(5):947-48.

3. P. Dutta, S. A. Khan, A. M. Khan, J. Borah, C. K. Sarmah \& J Mahanta. The effect of Insecticide-Treated mosquito Nets (ITMNs) on Japanese encephalitis virus sero conversion in Pigs \& Humans, American Journal of Tropical Medicine \& hygiene, vol.84, no.3, pp.466-472, 2011.

4. Solomon T, Dung NM, Kneen R, Gainsborough M, Vaughn DW, Khanh VT. Japanese encephalitis, J Neurol Neurosurg Psychiat 2000; 68: 405-415.

5. Potharaju Nagabhusana Rao, Japanese encephalitis, Indian paediatrics 2001; 38:1252-1264.

6. U K Misra, J Kalita. Prognosis of Japanese encephalitis patients with dystonia compared to those with parkinsonian features only Postgrad Med J2002; 78:238241.

7. Kumar R, Mathur A, Singh KB, Sitholey P, Prasad M, Shukla $\mathrm{R}$ et al. Clinical sequelae of Japanese encephalitis in children. Indian J Med Res 1993; 97: 9-13.

8. T.Solomon, T. T. Tho, P. Lewthwaite et al. A cohort study to assess the new WHO Japanese encephalitis surveillance standards, Bulletin of the World Health Organization, Vol.86, no.3, pp.178-186, 2008.

9. A Rayamajhi, R. Singh, R. Prasad, B. Khanal \& S. Singhi, Study of Japanese encephalitis \& other viral encephalitis in Nepali children. Paediatrics International, vol. 49, no.6, pp.978-984, 2007.

10. Osborn Anne G. Infections of the brain \& it's linings. In Diagnostic Neuroradiology, St Luise, Mosby -year book; 1994:673-715.
11. Sundar Twayana Ram, Ami Neuberger, Lekh Jung Thapa, Rana Pramendra Vir Singh, BenShofty, Eli Schwartz. Japanese Encephalitis among patients with acute Encephalitic Syndrome admitted to a tertiary Hospital in Chitwan, Nepal -A prospective Observational Study. PLoS ONE 9 (6):e99999.doi:10.1371/journal. pone 0099999.June 2014/ volume 9/ issue 6/e99999.

12. P. G. E. Kennedy Viral encephalitis: causes, differential diagnosis, and management, J Neurol Neurosurg Psychiatry 2004; 75 (suppll):i10-i15.doi:10.1136/jnnp.2003.034280.

13. Naoki Kawamura et al An Update on Diagnostic Imaging Studies for Viral Encephalitis Future Virology 2012; 7(9):901-909.

14. Lakshya J Basumatary, D. Raja Dipu Bhuyan, Marami Das, Munindra Goswami, Ashok K Kayal. Clinical \& Radiological spectrum of Japanese Encephalitis. Journal of the Neurological Sciences: 325 (2013) 15-21.

15. Kalita J, Misra UK. Comparison of CT scan and MRI findings in the diagnosis of Japanese encephalitis. J Neurol Sci2000; 174:3-8.

16. Kumar S, Misra UK, Kalita J, et al.MRI in Japanese encephalitis.Neuroradiology1997; 39:180-84.

17. Shoji $H$, Kida H, Hino $H$, et al. Magnetic resonance imaging findings in Japanese encephalitis. White matter lesions. J Neuroimaging1994; 4:206-11.

18. Misra UK, Kalita J, Jain SK, Mathur A. Radiological and neurophysiological changes in Japanese encephalitis. J Neurol Neurosurg Psychiatry.1994; 57:1484-1487.

19. Maschke $M$, et al. Update on neuroimaging in infectious central nervous system disease. Curr. Opin. Neurol. 17(4), 475-480 (2004).

20. $\mathrm{T}$ suchiya $\mathrm{K}$, et al Diffusion-weighted MR imaging of encephalitis. Am. J. Roentgenol. 173(4), 1097-1099 (1999).

21. White ML, Edwards-Brown MK. Fluid attenuated inversion recovery (FLAIR), MRI of herpes encephalitis. J Comput Assist Tomogr 1995; 19:501-505.

22. Kiroglu Y, Calli C, Yunten N, et al. Diffusion-weighted MR imaging of viral encephalitis. Neuroradiology 2006; 48:875880.

23. Prakash M, et al. Diffusion-weighted MR imaging in Japanese encephalitis. J. Comput. Assist. Tomogr. 28(6), 756-761 (2004).

24. S.K. Handique et al. Coinfection of Japanese Encephalitis with Neurocysticercosis: An Imaging Study, October 10, 2007 as 10.3174/ajnr. A0769.

25. Singh P, Kalra N, Ratho R. K. Shankar S Khandelwal N, Suri S. Co-existent Neurocysticerci \& Japanese B. Encephalitis MR Imaging correlation. AJNR 2001, 22:1131-6.

26. Kalita J, Misra UK, Pandey S, et al. A comparison of clinical $\&$ radiological findings in adults \& children with Japanese Encephalitis. Arch Neurol2003; 60:1760-64. 
Fig. 1(A-C): Bilateral asymmetrical T2/FLAIR hyperintense areas (White arrows in Fig: 1A \& C) in bilateral thalami \& Substantia nigra. T2 central hyper and peripheral hypointense and T1 peripheral hyperintense rim like lesion with mild perilesional edema in left frontal lobe showing eccentrically located calcified scolex (Red \& White arrow in Fig: 1A) , on post contrast scan peripheral rim like enhancement \& irregular gyriform pattern of leptomeningeal enhancement (Fig: 1D).

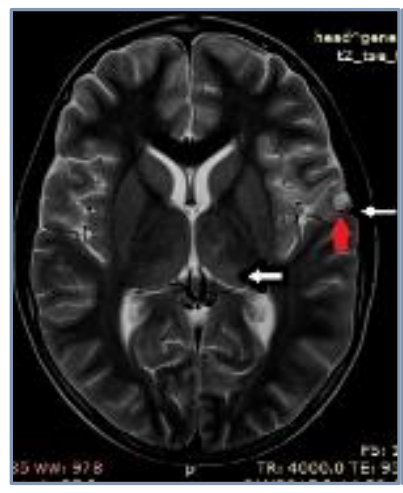

Fig. 1A

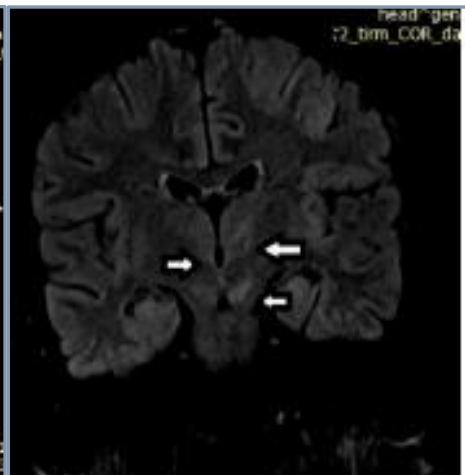

Fig. 1B

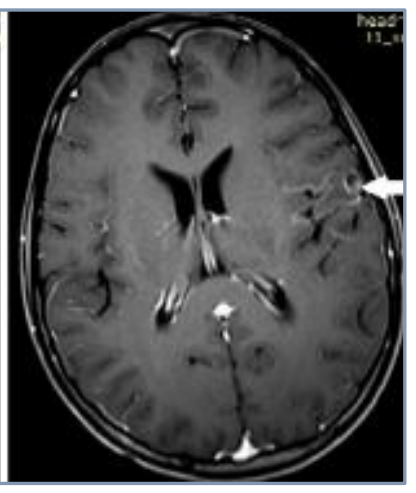

Fig. 1C

Fig. 2(A-D): Symmetrical T2/FLAIR hyperintensities in bilateral Gangliothalamic regions and bilateral Substantia nigra (White arrows in Fig. 2A \& B) which shows restriction on DWI with variable but predominantly high ADC values (Fig. 2C \& D white arrow). Thin walled T1 hypo and T2 hyperintense lesion with peripheral T2 hypointense rim and T2 hypointense eccentric scolex (Red arrow in Fig. A \& B ) in the left posterior parietal lobe with minimal perilesional edema without post contrast enhancement (Red arrow in Fig. E).

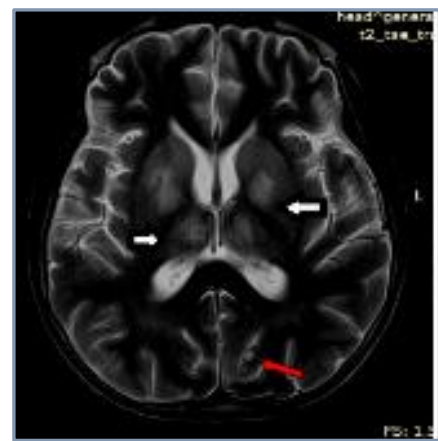

Fig. $2 A$

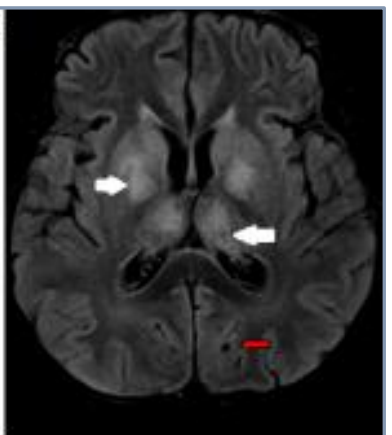

Fig. $2 B$

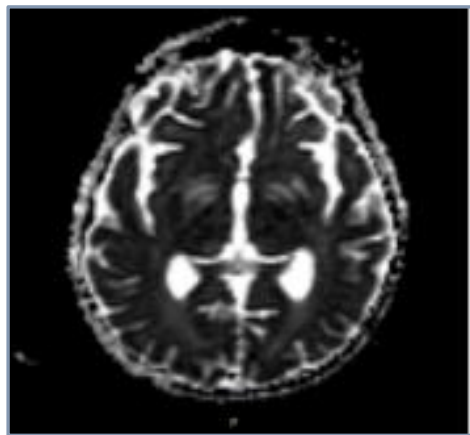

Fig. $2 C$

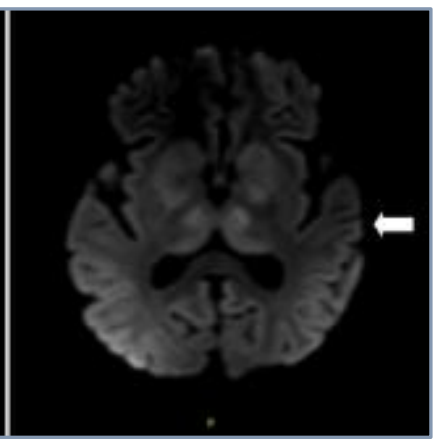

Fig. 2D

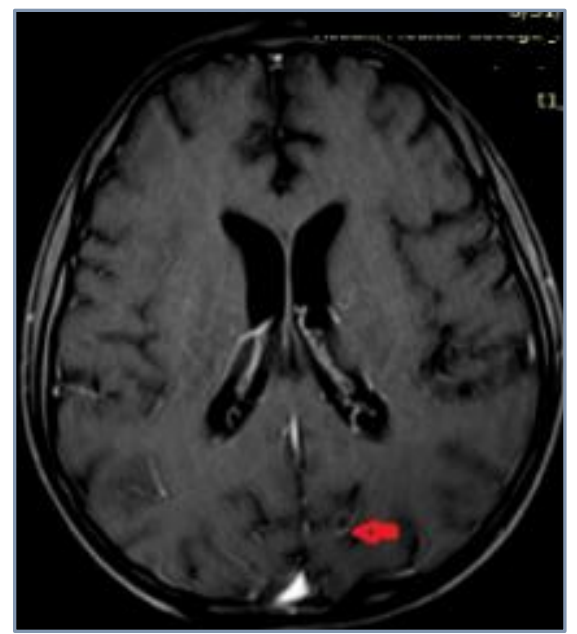

Fig. $2 E$ 
Fig. 3: Asymmetrical T2/FLAIR hyperintensities in bilateral ganglio-thalamic regions (White stars in) and anterior paramedian part of right frontal lobe (Red star).

Fig. 4: FLAIR hyperintense signals in the bilateral thalamus (Red stars) and left insular cortex (Red arrow).

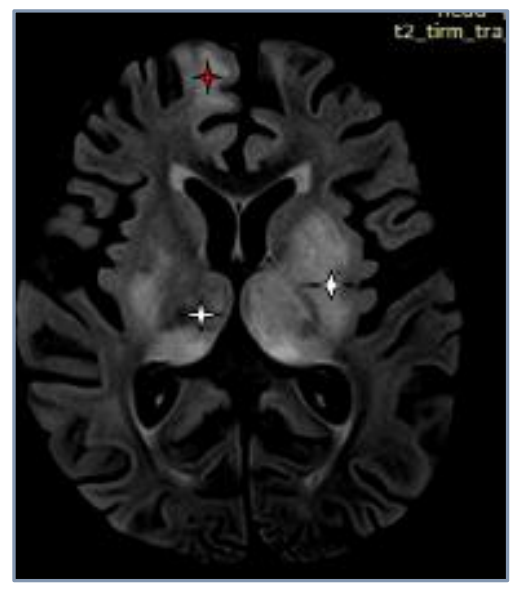

Fig. 3

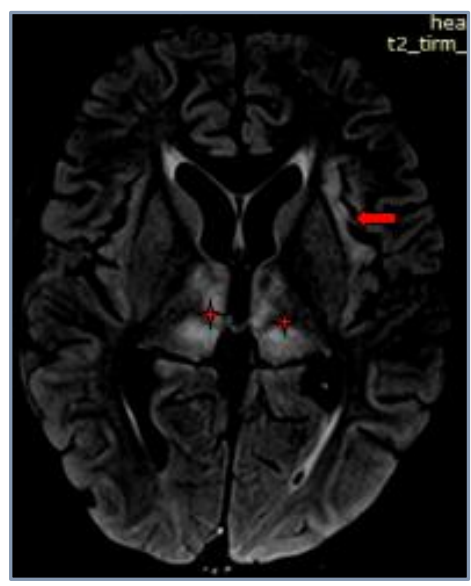

Fig. 4

Fig. 5 A-C: Coronal FLAIR (A) sequences showing bilateral asymmetrical hyperintensities in the Thalamus (White stars) \& Substantia nigra (black arrows) with Hippocampal (B red star) \& right insular cortex (D white arrow) involvement. T2 peripheral hypo and central fluid density lesion showing blooming on GRE representing calcified scolex of cysticercus in the right Occipital lobe with perilesional edema(5 C)

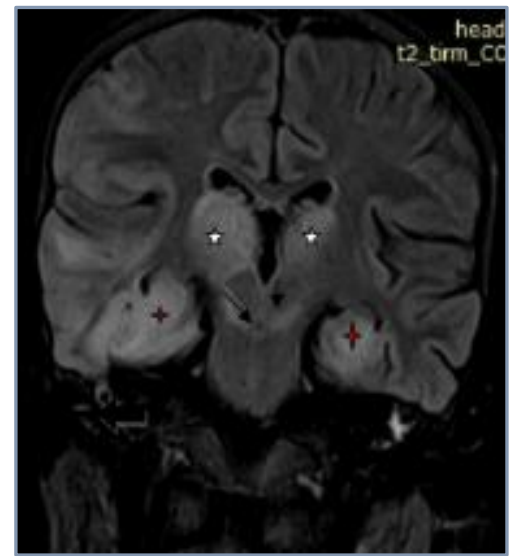

Fig. 5A

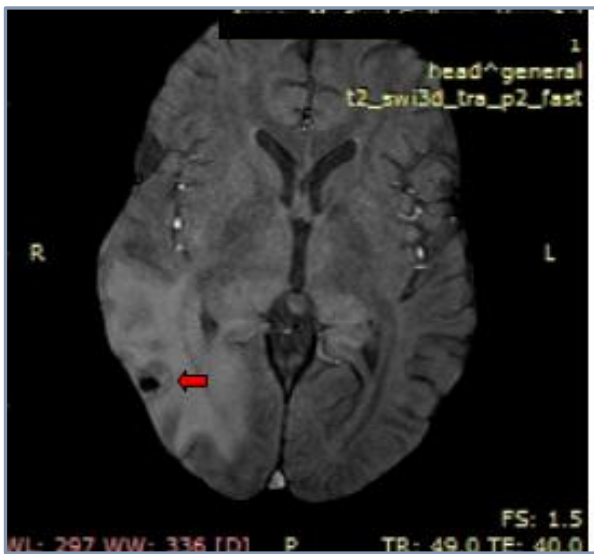

Fig. 5B

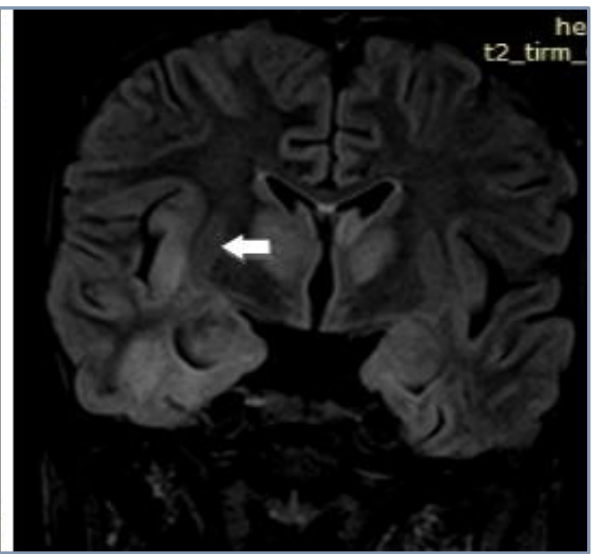

Fig. 5C 